\title{
ESTUDIO FLORÍSTICO, FENOLÓGICO, AUTOECOLÓGICO Y FITOGEOGRÁFICO DEL MACROFITOBENTOS DE LA Mar Chica (Sebcha Buareg de Nador, MEDITERRÁNEO MARROQUí)
}

\author{
Juan Antonio GONZÁLEZ \& Francisco CONDE
}

RESUMEN: El presente trabajo, es el primer estudio fitobentónico sobre la Sebcha Buareg (Nador, Marruecos mediterráneo), laguna litoral hipersalina. Aportamos una lista de 113 taxa (62 Rhodophyceae, 18 Phaeophyceae, 31 Chlorophyceae y 2 Liliopsida). Se completa el trabajo con datos fenológicos, autoecológicos y fitogeográficos de los taxa citados.

Palabras clave: Algas bentónicas marinas, laguna litoral, Marruecos, Mediterráneo.

SUMMARY: This labour is the first fitobentonic study about the "Sebcha Buareg" (Nador, Mediterranean Morocco), hypersaline lagoon. We contribute a list over 113 species (62 Rhodophyceae, 18 Phaeophyceae, 31 Chlorophyceae y 2 Liliopsida). It's completed the labour with fenological, autoecological and fitogeographical facts of the aforementioned species.

Key words: Benthic marine algae, lagoon, Morocco, Mediterranean Sea.

\section{INTRODUCCIÓN}

La Mar Chica (Sebcha Buareg de Nador) está situada en el noreste de Marruecos, a orillas del Mar Mediterráneo y muy próxima, en su extremo noroeste, a la ciudad española de Melilla.

Se trata de una albufera hipersalina, separada del resto del Mar Mediterráneo por una barra arenosa de unos $24 \mathrm{Km}$ de longitud y unos $250 \mathrm{~m}$ de anchura máxima. Actualmente este cordón arenoso sólo queda interrumpido por una pequeña bocana situada,más o menos, en su centro que tiene unos $40 \mathrm{~m}$ de anchura y 2,5 $\mathrm{m}$ de profundidad máxima. La superficie total es de unos $114 \mathrm{Km}^{2}$ (Erimesco 1961),y las profundidades máximas, medidas para el presente trabajo, sobrepasan escasamente los $7 \mathrm{~m}$ en su zona central (Mapa 1). 


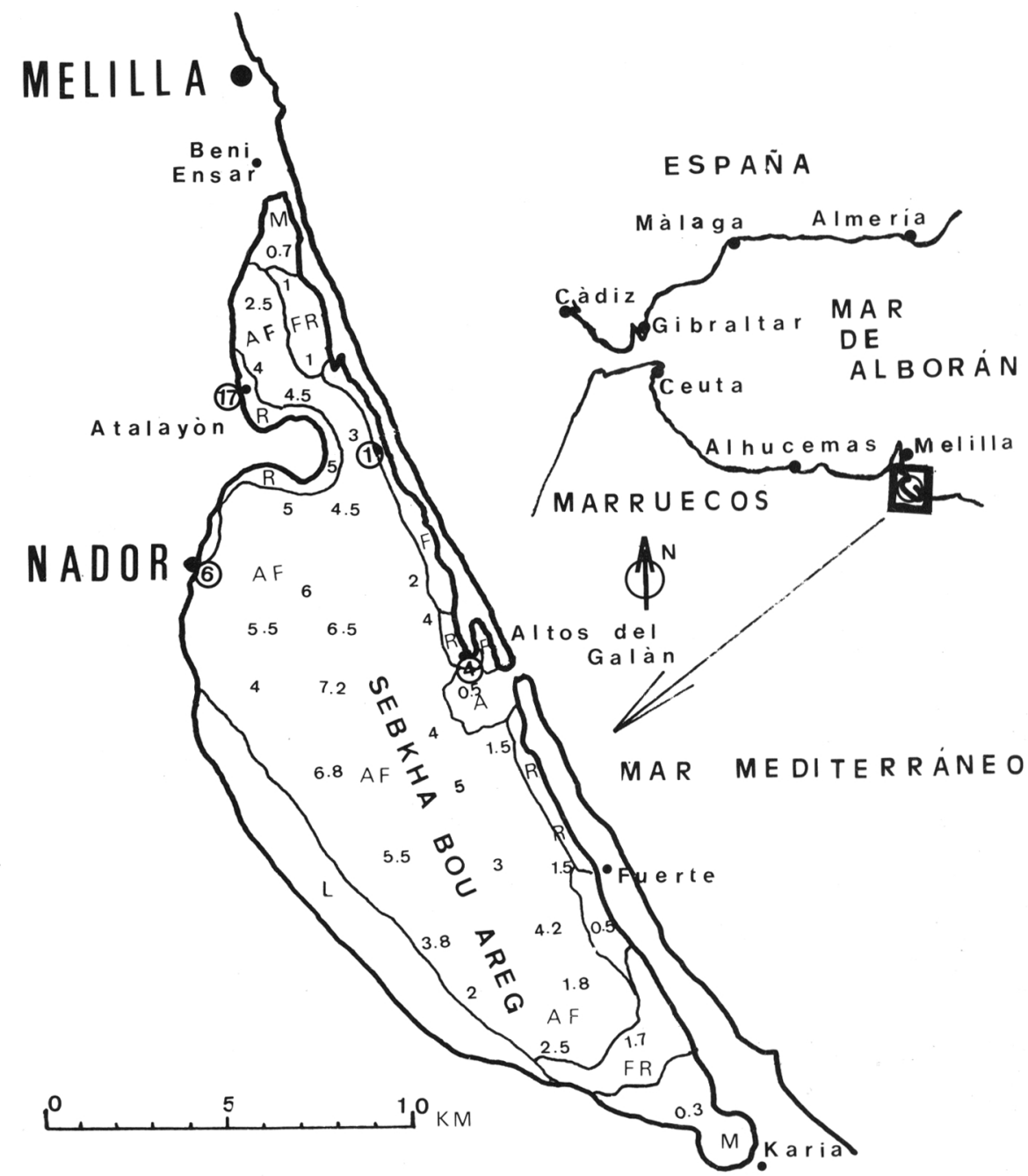

Mapa 1. Situación geográfica de las localidades de muestreo (rodeadas por el círculo: 1, 4, 6 y 17). Los números más pequeños, corresponden a mediciones de profundidades, en metros, de otras estaciones de muestreo. Las letras corresponden a la naturaleza del fondo (A: arena, F:fango, AF: arena fangosa, FR: fango reductor, L: limo, R: rocas y M: marismas).

Predominan los fondos arenoso-fangosos, que ocupan la práctica totalidad de la superficie, llegando a un máximo de degradación en determinadas zonas y en los extremos geográficos de la albufera donde toman aspecto de marisma. La costa es baja y arenosa, si exceptuamos la elevación volcánica de El Atalayón y sus alrededores, en la orilla interna, y las pequeñas alturas areniscosas de los Altos del Galán en la misma barra arenosa. 
Los datos más recientes sobre parámetros físico-químicos nos los proporcionan los estudios iniciales realizados por la Empresa Marost de cultivos marinos (1987), que en la actualidad opera en la Mar Chica en la producción de ostras, almejas y langostinos. Estos datos ratifican, en gran medida, los anteriores de Lozano Cabo (1953). Las salinidades se encuentran algo elevadas sobre las del Mediterráneo, sobrepasando el $40 \%$ al final del verano. El pH es ligeramente básico, oscilando entre 8,2 y 8,4 . El análisis de las temperaturas medias mensuales muestra que en verano las aguas lagunares son más calientes que las del mar y en invierno más frías. Las temperaturas máximas corresponden a los $29^{\circ} \mathrm{C}$ registrados en el extremo noroeste en el mes de agosto y las mínimas a los $9,4^{\circ} \mathrm{C}$ del mes de enero cerca del extremo sureste. Las máximas concuerdan con los datos recogidos por nosotros los días de muestreo, pero no así las mínimas que no bajaron en estos días de $13,4^{\circ} \mathrm{C}$.

En la Mar Chica, donde han predominado estudios hidrológicos y sobre recursos pesqueros, sólo hay citas muy puntuales sobre fitobentos. Cabe destacar a Lozano Cabo (op.cit.),que da 2 especies de fanerágamas: Posidonia oceanica y Zostera marina; a Sauvageau y R. de Buen (in Hamel 1931-1939) que citan cerca de El Atalayón la especie Cystoseira tingitana; y ya más recientemente a Yus \& Cabo (1985) en un trabajo naturalístico general, que toman de Lozano las dos especies de fanerágamas y dan una nueva especie: Enteromorpha compressa.

\section{MATERIAL Y MÉTODOS}

El trabajo que presentamos es el fruto de las recogidas de datos y material realizadas desde el mes de mayo de 1989 a octubre de 1990.

En principio se realizó un muestreo exhaustivo por toda la superficie de la albufera, en el que se llegaron a establecer 36 estaciones (Mapa 1). Posteriormente, y dada la gran uniformidad que presentaban muchas de ellas, elegimos sólo 4, que en conjunto nos dan una idea global bastante exacta de lo que es la laguna. Dos de estas estaciones ( 17 y 4) corresponden a las dos zonas rocosas ya citadas (Atalayón y Altos del Galán). Una tercera (6) se situó en el antiguo puerto pesquero de Nador, con características propias, sobre todo en cuanto al nivel de degradación de su costa. Y la cuarta (1) corresponde a un transecto realizado desde la orilla hasta unos $3 \mathrm{~m}$ de profundidad en una zona de la barra que puede considerarse representativa de la casi totalidad de ella.

La toma de datos y la recolección se hicieron según la metodología clásica. El catálogo florístico se ha confeccionado utilizando fundamentalmente los criterios nomenclaturales que siguen Athanasiadis (1987) y Boudouresque \& Perret (1987). La ordenación sistemática de los taxa sigue casi siempre los criterios de Wynne \& Kraft (1981) para los rodófitos (pero segregando el orden Corallinales del Cryptonemiales en base a Silva \& Johansen,1986) y los clorófitos,y los de Parke \& Dixon (1976) para los feófitos. 
Gran parte del material recolectado fue depositado en el Herbario del Departamento de Biología Vegetal de la Universidad de Málaga (Phyc MGC).

Para cada taxon del catálogo florístico se indica: a) Estación de recolección (según mapa1), seguida entre paréntesis de los meses de recolección expresados en números romanos. Tras cada estación aparece el índice de abundancia y grado de cobertura de Braun-Blanquet y Pavillard. b) Autoecología de los taxa asignándolos a grupos ecológicos según la terminología definida en Boudouresque (1984), seguida del elemento fitogeográfico al que pertenece según Giaconne et al. (1985). c) Otros datos relativos a presencia de elementos reproductores, epifitismo, difusión, etc.

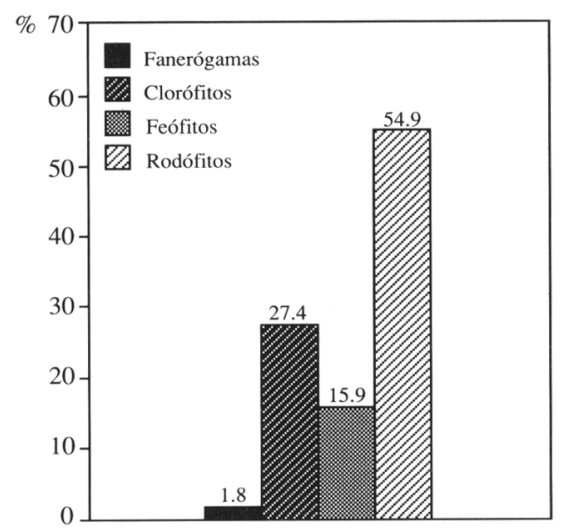

Fig. 1. Porcentaje del número de taxa catalogados.

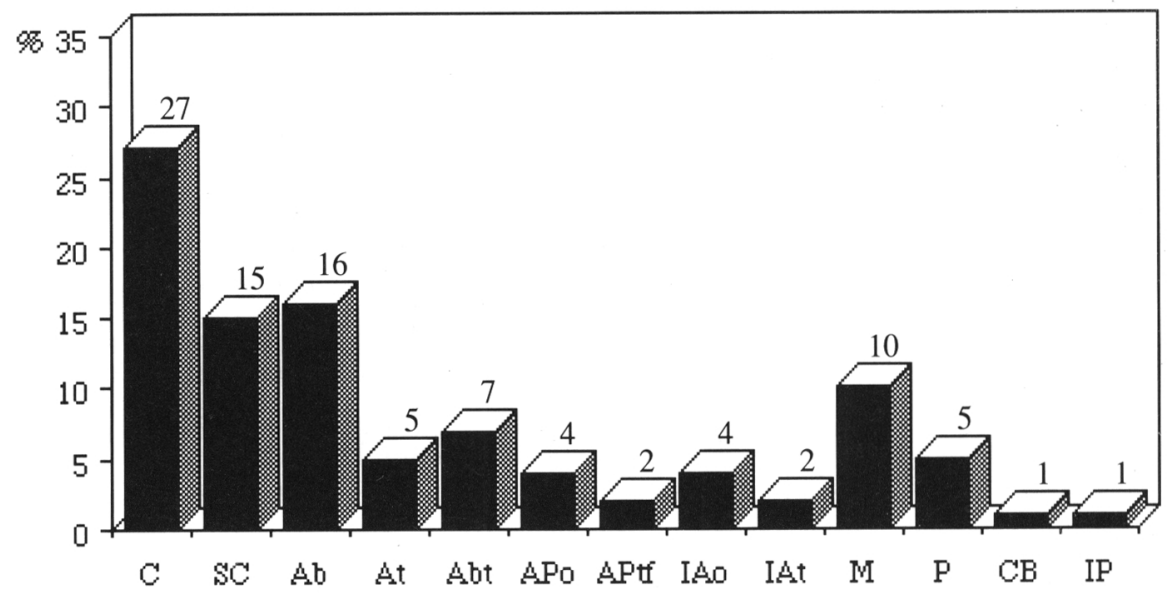

Fig. 2. Porcentaje del origen fitogeográfico de los taxa (sensu Giaccone \& al., 1985). 


\title{
CATÁLOGO FLORÍSTICO
}

\author{
RHODOPHYCEAE
}

\author{
NEMALIALES
}

Acrochaetiaceae

Audouinella codii (Crouan) Garbary

a) $4(\mathrm{VI})+$. b) PhI, Ab. c) Monósporas (VI). Sobre Sargassum vulgare

Audouinella daviesii (Dillwyn) Woelkerling

a) 4 (VI a IX) 1; 17 (V,VI,IX,X,XI) 1. b) ISR, APo. c) Monósporas (V a XI). Siempre epífita, sobre Cystoseira crinita, Cystoseira compressa, Gelidium latifolium, Sargassum vulgare, etc.

Audouinella moniliformis (Rosenvinge) Garbary

a) 17 (VI, VIII) +. b) ISR, M. c) Epífita de Cladophora echinus

Audouinella parvula (Kylin) Dixon

a) 17 (VI, VIII) 1. b) PhI, Ab. c) Monósporas(VI, VIII). Epífita de Cystoseira crinita y C. compressa.

Audouinella saviana (Meneghini) Woelkerling

a) 4 (VI,VII,X) 1; 17 (VI,VIII,IX,X) 1. b) PhI, Abt. c) Monósporas (VI,VIII,IX,X). Epífita de Sargassum vulgare, Cystoseira sp.pl., Cladophora sp.pl.

\section{Gelidiaceae}

Gelidium latifolium (Grev.) Born. et Thur.

a) 4 (I a XII) 4. b) PhI, SC.c) Forma una franja continua entre 0 y $50 \mathrm{~cm}$ de profundidad en el cantil vertical, asociada a otras especies como Hypnea musciformis y Caulacanthus ustulatus. Forófita de Cladophoraceae, Jania rubens, Ceramium diaphanum.

Gelidium pusillum (Stackhouse) Le Jolis

a) 4 (I a XII) 4; 6 (I a XII) 4;17 (I a XII) 3. b) PhIB, C. c) Cistocarpos (V,VI). Tetrasporangios (IV,V,VI,X,XI). Forma en muchos lugares una franja más o menos continua en el nivel superior.

\section{GIGARTINALES}

\section{Hypneaceae}

Hypnea musciformis (Wulfen) Lamouroux

a) 1 (I a XII) 2; 4(I a XII) 3; 6 (I a XII) 4; 17 (I a XII) 3. b) PhIT, P. c) Tetrasporocistos (V a XI). Enredada a Cystoseira crinita en fondos arenosos. Forófita de Griffithsia tenuis, Ceramium flaccidum, Jania rubens. Especie extraordinariamente abundante y difundida por 
toda la costa a menos de $2 \mathrm{~m}$ de profundidad. Mayor desarrollo vegetativo en lugares de alta degradación. En la estación 6 (Puerto de Nador) cubre el fondo asociada a Caulerpa prolifera.

\section{Caulacanthaceae}

Caulacanthus ustulatus (Mertens) Kützing

a) 4 (V,VI,VIII,IX,X) 1; 6 (V,IX,XI) 1; 17(III,VIII a XI)1.b) SI, SC. c) Tetrasporangios (VI,X,XI). Enredada a otras algas como Sargassum vulgare e Hypnea musciformis.

\section{Gracilariaceae}

Gracilaria armata (C.Agardh) J.Agardh
a) 17 (VIII) +. b) PhIB, At

Gracilaria bursa-pastoris (S.G.Gmel.) Silva

a) $1(\mathrm{~V}, \mathrm{VI})+$. b) PhI, SC. c) En fondos de fango reductor recubierto al $100 \%$ de Caulerpa prolifera, entre 70 y $150 \mathrm{~cm}$ de profundidad. Asociada además a Valonia utricularis, Zostera marina y Zostera noltii.

Gracilaria cervicornis (Turner) J.Agardh
a) $17(\mathrm{VIII}, \mathrm{IX}, \mathrm{X})+$. b) PhI,-

Gracilaria verrucosa (Hudson) Papenfuss

a) 3 (VII) +; 6(I a XII) 2; 17 (I a XII) 2. b) PhI, C. c) Cistocarpos (VI,I,II). Especialmente difundida en la orilla interna de la laguna, con alto grado de polución, asociada a Ulva olivascens, Enteromorpha intestinalis e Hypnea musciformis. Forófita de Ceramium diaphanum.

\section{Phyllophoraceae}

Gymnogongrus griffithsiae (Turner) Martens

a) 17 (XII,I,II,VII,VIII) +. b) FM, CB. c) Ejemplares sueltos. Muy restringida a la escollera del antiguo lavadero del mineral en El Atalayón.

Phyllophora crispa (Hudson) Dixon

a) 4 (VI)+. b) SI, Ab. c) Sólo se ha recolectado un ejemplar con Jania rubens de epífito.

Schottera nicaensis (Lamouroux) Guiry et Hollenberg

a) 4 (I a XII) 2. b) SSB, Ab. c) Muy restringida bajo cornisas y en oquedades, asociada a Peyssonelia squamaria y Bryopsis plumosa.Tetrásporas (X).

\section{Gigartinaceae}

Gigartina acicularis (Roth) Lamouroux

a) 4 (I a XII) 3; 6 (I a XII) 3; 17 (I a XII) 2. b) PhIP, C. c) Cistocarpos (XII,I,II). Enmarañada a otras especies a escasa profundidad. Forófita de Centroceras clavulatum. 


\section{CORALLINALES}

\section{Corallinaceae}

Amphiroa beauvoisii Lamouroux

a) 6 (IX a II) 2. b) SC, IAt. c) Conceptáculos (IX,X). Sólo aparece en la escollera del puerto a menos de $50 \mathrm{~cm}$ de profundidad. Forófita de Antithamnionella elegans y Centroceras clavulatum. Epífita en la base de Ulva olivascens.

Fosliella farinosa (Lamouroux) Howe

a) 1 (I a XII) 1; 4 (I a XII) 1; 6 (I a XII) 1 ; 17 (I a XII) 1. b) ISR, C. c) Conceptáculos (V,VI,X,XI). Muy difundida, epifitando a Zostera marina, por todas las zonas a menos de 2,5 $\mathrm{m}$ de profundidad.

Jania rubens (L.) Lamouroux

a) 4 (I a XII) 4; 6 (I a XII) 3; 17 (I a XII) 4. b) PhI, C. c) Conceptáculos (VII a X). Dominando sobre rocas horizontales muy iluminadas, asociada a Laurencia obtusa. Epífita de otras muchas.

Schmitziella endophloea Born. et Batters

a) 4 (VI,VII,IX) +. b) AS, C. c) Siempre epífita de Cladophora prolifera.

Spongites fruticulosa Kützing

a) 4 (I a XII) 1; 17 (I a XII) 1. b) SM, Ab. c) Muy común sobre el gasterópodo Thais haemastoma

Titanoderma pustullatum (Lamouroux) Nägeli

a) 4 (I a XII) 1; 17 (I a XII) 1. b) ISR, C. c) Epífita de Gelidium latifolium,Sargassum vulgare, Cystoseira sp.pl.

\section{CRYPTONEMIALES}

Cryptonemiaceae

Grateloupia filicina C. Agardh

a) $17(\mathrm{IV}, \mathrm{V})+$. b) SSBc, SC. c) Tetrásporas (IV).

Peyssoneliaceae

Peyssonelia bornetti Bouderesque et Denizot

a) 4 (X)+. b) SC, M. c) Nematecios (X)

Peyssonelia dubyi P.L. et H.M. Crouan

a) 4 (I a XII) 1; 6 (I a XII) 1; 17 (I a XII) 1. b) ISR, Ab. c) Nematecios (IV,V). Común sobre moluscos. 
Peyssonelia squamaria (Gmel.) Dcne.

a) 4 (I a XII) 2. b) SCIt, M. c) Nematecios (IV a VIII). Confinada a lugares esciáfilos, asociándose a Schottera nicaensis, Bryopsis plumosa y Colpomenia sinuosa.

\section{RHODYMENIALES}

Champiaceae

Gastroclonium clavatum (Roth) Ardissone

a) $4(\mathrm{IV}, \mathrm{V}, \mathrm{VI})+$. b) EM, M.

\section{CERAMIALES}

\section{Ceramiaceae}

Antithamnion cruciatum (C.Agardh) Nägeli

a) $4(\mathrm{IV}, \mathrm{V})+$. b) ISR, Abt. c) Tetrásporas (V). Epífita de Gelidium latifolium.

Antithamnionella elegans (Berthold) Boudouresque et Verlaque

a) 4 (VII,VIII) +; 6(XII,I,II) 1. b) SSBf, M. c) Tetrásporas (VII,VIII). Epífita de Amphiroa beauvoisii.

Callithamnion neglectum (G. Feldmann-Mazoyer) Ballesteros et Romero.

a) 4(IV) +. b) SC, At. c) Epífita de Gelidium pusillum.

Centroceras clavulatum Montagne

a) 4 (I a XII) 1; 6 (I a XII) 2; 17 (I a XII) 1. b) PhIP, C. c) Máximo desarrollo vegetativo en estaciones muy contaminadas, asociada a Gigartina acicularis, Amphiroa beauvoisii e Hypnea musciformis.

Ceramium cingulatum A. Weber

a) $4(\mathrm{IV}, \mathrm{V})+$. b) -. c) Sobre Cystoseira crinita y Sargassum vulgare.

Ceramium diaphanum (Lighfoot) Roth

a) 4 (X) +; 17 (IV) +. b) ISR, SC. c) Tetrásporas (X). Sobre Laurencia obtusa y Gracilaria verrucosa.

Ceramium flaccidum (Harvey) Ardissone

a) 1 (VII) +; 4 (V,VII,VIII,X) +; 17 (V a II) +. b) ISR, C. c) Sobre Cystoseira crinita, Sargassum vulgare, Zostera marina..

Ceramium rubrum (Hudson) C. Agardh

a) 4 (V,VI,XII,I)+. b) PhIB, APo. c) Tetrásporas (VI). Sobre hidrozoos, Padina pavonica y Zostera marina.

Ceramium tenerrimum (Martens) Okamura

a) 4 (IV) +. b) PhI, SC. c) Epífita de Alsidium corallinum. 
Ceramium tenuissimum (Roth)J. Agardh

a) 1 (VI,VII) +; 4 (V,VI,XII) 1; 17 (XI,XII,I,II) 1. b) PhIC, SC. c) Sobre Zostera marina y Cystoseira sp.pl.

Griffithsia tenuis C. Agardh

a) 4 (IV,V,VIII) +; 17 (V,VIII) +. b) -, M. c) Tetrasporangios (IV,V,VIII). Sobre Hypnea musciformis

Monosporus pedicellatus (Smith) Solier var. tenuis Mazoyer

a) $17($ III,IV,V) +. b) SIC, M

Spyridia filamentosa (Wulfen) Harvey

a) 1 (I a XII) 2; 4 (I a XII) 2; 6 (I a XII) 1; 17 (I a XII) 2. b) PhIT, C. c) Tetrasporófitos

(X,XI). Sobre Cystoseira crinita. Forófita de Enteromorpha multiramosa y Cladophora sp.pl. Muy difundida por toda la albufera, especialmente en fondos arenoso-fangosos, fija a guijarros sueltos o a valvas vacías de moluscos. Siempre a menos de $2 \mathrm{~m}$ de profundidad.

Dasyaceae

Dasya baillouviana (Gmelin) Montagne

a) $4($ IV,V)+. b) IAo. c) Estiquidios (IV,V). Epífita de Gelidium pusillum.

Heterosiphonia crispella (C.Agardh) Wynne

a) $4(\mathrm{~V}, \mathrm{VI})+$. b) SI, SC

\section{Delesseriaceae}

Acrosorium uncinatum (Turner) Kylin

a) $4(\mathrm{IV}, \mathrm{V}, \mathrm{VI})+$. b) SI, SC. c) Tetrasporocistos (IV,V). Sobre Bryopsis plumosa y Schottera nicaensis.

Rhodomelaceae

Alsidium corallinum C. Agardh

a) 4 (I a XII) 1; 6 (I a XII) 1; 17 (I a XII) 1. b) PhIT, At. c) Forófito de Cladophora dalmatica, Enteromorpha torta, E.flexuosa, Ceramium tenerrimum.

Chondria dasiphylla (Woodward) C. Agardh

a) $17(\mathrm{III}, \mathrm{IV}, \mathrm{V})+$. b) PhIC, SC. c) Tetrásporas (III,IV,V)

Chondria mairei J. Feldmann

a) $4(\mathrm{X})+$. b) HP, M. c) Tetrásporas y cistocarpos (X). Sobre Zostera marina.

Chondria tenuissima (Good. et Woodw.) C. Agardh

a) 1 (X a VI) 2; 17 (X a VII) 1. b) PhIC, IAo. c) Tetrasporocistos (XII a II). Cistocarpos (V,VI y X a II). Especialmente abundante y difundida como epífita de Zostera marina y Zostera noltii. 
Herposiphonia secunda (C. Agardh) Ambronn

a) 1 (V,VI) +; 4 (IV,V,VII,VIII) 1; 17 (V a IX y XII)1. b) PhIC, P. c) Sobre Cladophoraceae, Jania rubens, Gelidium pusillum.

Herposiphonia secunda (C. Ag.) Ambronn fma. tenella Wynne

a) 1 (IV,V,X,XI). b) PhIC, P. c) Sobre Gelidium pusillum y Zostera marina.

Laurencia obtusa (Hudson) Lamouroux

a) 4 (IV a XII) 2; 17 (VII a XII) 2. b) PhI, C. c) Tetrásporas (VIII,IX,X). Forófita de Blidingia marginata, Enteromorpha multiramosa, Ceramium diaphanum, entre otras. Muy difundida en rocas horizontales asociada a Jania rubens y Gelidium pusillum.

Laurencia pinnatifida (Hudson) Lamouroux

a) 4 (III a VI) 1; 17 (I a VII) 1. b) PhIB, SC. c) Cistocarpos (I,II)

Polysiphonia elongata (Hudson) Sprengel

a) 17 (III,IV,V,X,XI) +. b) CC, Abt. c) Cistocarpos (III,IV,V)

Polysiphonia fruticulosa (Wulfen) Sprengel

a) $4(\mathrm{X})+$. b) $\mathrm{PhIB}, \mathrm{Ab}$

Polysiphonia violacea (Roth) Greville

a) 6 (I a XII) 2; 17 (I a XII)2. b) RMM1, Ab. c) Tetrásporas (I a IV). Restringida a zonas rocosas degradadas donde abunda formando penachos de hasta $10 \mathrm{~cm}$

\section{PORPHYRIDIALES}

\section{Goniotrichaceae}

Stylonema alsidii (Zanardini) Drew vulgare.

a) 4 (X) +; 17(II) +. b) ISR, C. c) Sobre Cladophora sp., Gelidium latifolium y Sargassum

Stylonema cornu-cervi Reinsch multiramosa.

a) 4 (XII,I,II) +; 17 (XII,I,II,III) +. b) SI, APo. c) Sobre Cladophora sp. y Enteromorpha

\section{BANGIALES}

\section{Erythropeltidaceae}

Erythropeltis subintegra (Rosenvinge) Kornann et Sahling

a) 4 (VII) +. b) ETN, C. c) Sobre Gelidium latifolium.

Erythrotrichia carnea (Dillwyn) J. Agardh

a) 1 (I a XII) 1; 4 (I a XII) 1; 6 (I a XII) 1; 17 (I a XII) 1. b) ETN, C. c) Siempre epífita, sobre muchas algas mayores. 
Erythrotrichia investiens (Zanardini) Bornet

a) 4 (II,VI,VII) +; 17 (VI,VII) +. b) ETN, Ab. c) Siempre epifitando especies mayores

Erythrotrichia reflexa

a) 1 (IV,VI) +; 4 (II a VI) +; 17 (III a VI y XI). b) ETN, Ab. c) Siempre epífita

\section{Bangiaceae}

Bangia atropurpurea( Roth) C. Agardh

a) 1 (XI a II) 1.b) RMS, C c) Sobre Zostera marina y estacas en los parques de cultivos de langostinos.

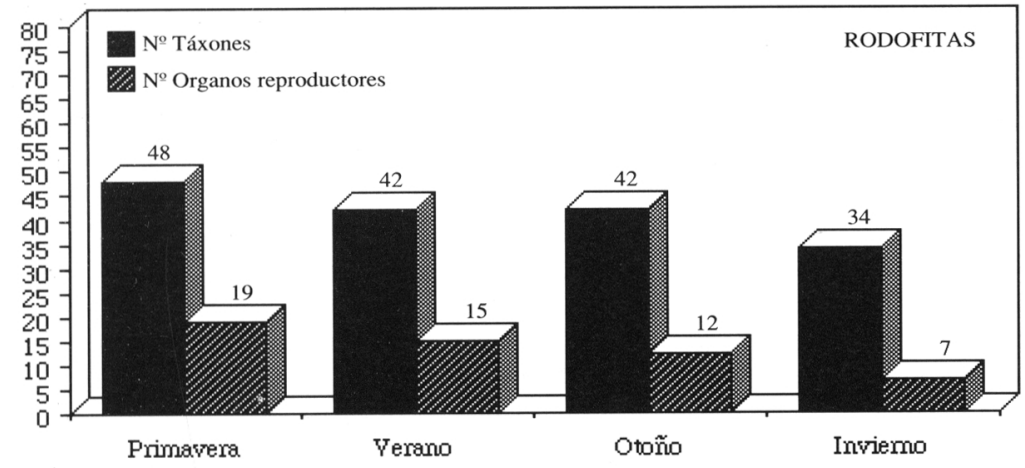

Fig. 3. Número total de rodófitas en las diferentes estaciones del año, junto con el número total de órganos reproductores en las distintas épocas anuales.

\section{PHAEOPHYCEAE}

\section{ECTOCARPALES}

\section{Ectocarpaceae}

Ectocarpus siliculosus (Dillwyn) Lyngbye

a) 17 (VI,VII,VIII) +. b) PhIP, SC. c) Esporangios pluriloculares (VI,VII,VIII). Sobre Cystoseira crinita

Feldmannia globifera (Kützing) Hamel

a) 1 (V,VI,X) +; 4 (I a XI) 1; 6 (VI,VII,IX,X) +; 17 (I a XII) 1. b) PhI, SC. c) Esporangios pluriloculares (V,VI,VII,IX,X,XI). Especialmente sobre Cystoseira sp.pl.

Feldmannia irregularis (Kützing) Hamel

a) 1 (VII,X) +; 4 (IX a II) 1. b) PhI, C. c) Esporangios uni y pluriloculares (XII a II) Sobre Zostera marina 
Giffordia mitchelliae (Harvey) Hamel

a) $17(\mathrm{~V}, \mathrm{VIII})+$. b) PhIP, C. c) Esporangios uni y pluriloculares (V,VIII). Sobre Cystoseira crinita.

Punctariaceae

Asperococcus turneri (Sm.) Hook.

a) $1(\mathrm{~V}, \mathrm{VI})$ 1; 4 (V,VI) 1. b) SRh, SC. c) Esporangios (V,VI)

Scytosiphonaceae

Colpomenia sinuosa (Roth) Derbés et Solier

a) 4 (V,VI,VII y XII a IV). b) PhIP, C. c) Esciáfila. Asociada bajo cornisas a Peyssonelia squamaria, Bryopsis plumosa y Schottera nicaensis

Scytosiphon lomentaria (Lyngbye) Link

a) 4 (V,VI) +; 17 (XII a II) 1. b) RMS, C. c) Ejemplares de hasta $15 \mathrm{~cm}$ en las zonas más degradadas.

\section{SPHACELARIALES}

Sphacelariaceae

Sphacelaria fusca (Hudson) S.F. Gray

a) 17 (VII,VIII) +. b) PhI, SC. c) Propágulos (VII,VIII)

Sphacelaria rigidula Kützing bornettii.

a) $4(\mathrm{X})+; 17(\mathrm{~V}, \mathrm{VI})+$. b) SSBc, SC. c) Propágulos (V,VI,X). Sobre Peyssonelia

\section{Stypocaulaceae}

Halopteris filicina (Grateloup) Kützing

a) 4 (I a XII) +; 17 (I a XII) +. b) PhI, APtf. c) Ejemplares muy sueltos, sin importancia en la biomasa total del ecosistema. No se han observado órganos reproductores.

Stypocaulon scoparium Kützing

a) $4(\mathrm{~V}$ a X $)+; 17(\mathrm{VI})+$. b) PhIC, SC. c) $\mathrm{Al}$ igual que la especie anterior, ejemplares muy sueltos.

\section{DICTYOTALES}

\section{Dictyotaceae}

Dictyota dichotoma (Hudson) Lamouroux

a) 4 (V) +; 17 (VI) +. b) PhIC, C. c) Sólo dos ejemplares. 
Padina pavonica (L.) Lamouroux

a) 4 (I a XII) 3; 17 (I a XII) 2. b) PhIC, P. c) Muy difundida en fondos rocosos horizontales bien iluminados. Forófita de Ceramium rubrum, Jania rubens y otras.

Taonia atomaria (Woodward) J. Agardh

a) $4(\mathrm{~V})+$. b) PhIC, Abt. c) En lugares algo esciáfilos. Sólo dos ejemplares, uno llevaba epífitas a Titanoderma pustullatum y Valonia utricularis.

Zonaria tournefortii (Lamour.) Montagne

a) 4 (VI) +. b) SCI, At. c) Sólo un ejemplar, bajo cornisa

\section{FUCALES}

Cystoseiraceae

Cystoseira compressa (Esper) Gerloff et Nizamuddin

a) 1 (I a XII) 1; 4 (I a XII) 3; 17 (I a XII) 2. b) PhIC, Ab. c) Receptáculos (I a VII). Con muchos epífitos: Audouinella sp.pl., Titanoderma pustullatum, Ceramium sp.pl., Enteromorpha sp.pl., Cladophora sp.pl., etc. Máximo de vegetación en primavera-verano.

Cystoseira crinita (Desfontaines) Bory

a) 1 (I a XII) 2; 4 (I a XII) 3; 17 (I a XII) 3. b) PhIC, M. c) Receptáculos (I a VII). Muy difundida incluso en fondos arenosos, aprovechando valvas vacías o guijarros para fijarse. Gran importancia en la biomasa total del ecosistema, junto con la especie anterior, con la que cohabita hasta los $2 \mathrm{~m}$ de profundidad. Multitud de epífitos.

Sargassaceae

Sargassum vulgare C. Agardh

a) 4 (IV a XI) 2. b) PhIC, P. c) Receptáculos (V,VI). Muchos epífitos de pequeña talla, además de Caulacanthus ustulatus y Alsidium corallinum.

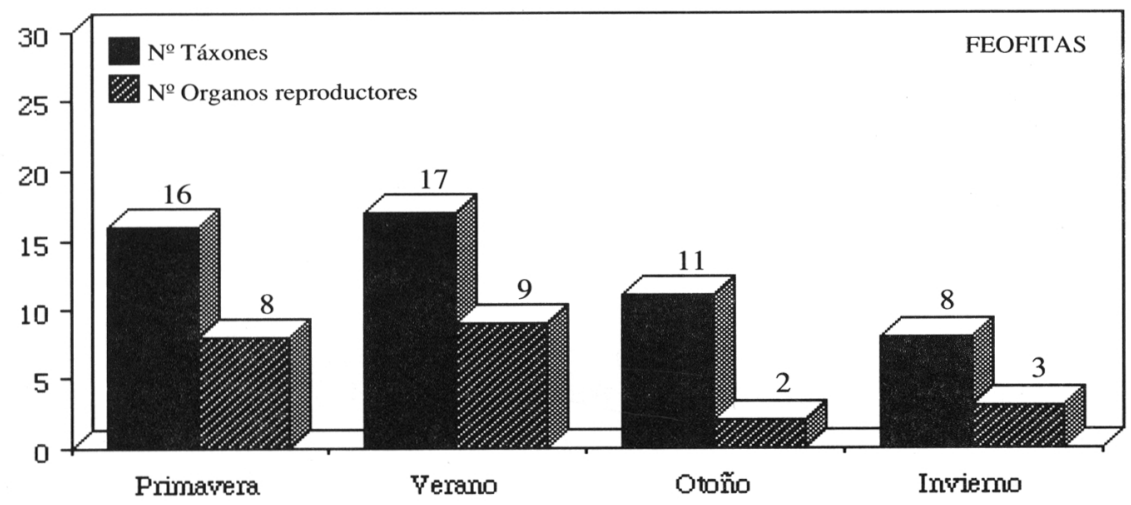

Fig. 4. Número total de feófitas en las diferentes estaciones del año, junto con el número total de órganos reproductores en las distintas épocas anuales. 


\section{CHLOROPHYCEAE}

\section{ULVALES}

Ulvaceae

Blidingia marginata (J. Agardh) Dangeard

a) $17(\mathrm{VI}, \mathrm{X})+$. b) $\mathrm{PhI}, \mathrm{Ab}$.

Enteromorpha clathrata (Roth) Greville

a) $17(\mathrm{IV}, \mathrm{V})+$. b),$- \mathrm{C}$

Enteromorpha compressa (L.) Greville

a) 1(I a XII)1;6(I a XII)1;17(I a XII)1. b) RMM2, C. c) Ampliamente difundida por toda la orilla interna.

Enteromorpha flexuosa (Wulfen) J. Agardh

a) 4 (I a XII) 1; 17 (I a XII) 1. b) PhIP, C

Enteromorpha intestinalis (L.) Link

a) 1 (I a XII) +; 6 (I a XII) 3; 17 (I a XII) 1. b) PhIP, C. c) Especialmente abundante en zonas muy degradadas asociada a Gracilaria verrucosa, Hypnea musciformis, Ulva olivascens y otras Ulváceas.

Enteromorpha linza (L.) J .Agardh

a) 1 (I a XII) +. b) PhIC, C. c) Aunque se da durante todo el año, sólo aparecen ejemplares sueltos en la misma orilla de la barra arenosa.

Enteromorpha multiramosa Bliding

a) 4 (I a XII) 2; 6 (I a XII) 2; 17 (I a XII) 3. b) PhIC, M. c) Epífita sobre Taonia atomaria, Hypnea musciformis, Spyridia filamentosa,etc.

Enteromorpha prolifera (Müller) J. Agardh

a) 1 (I a XII) 1; 4 (I a XII) 1; 6 (I a XII) 1; 17 (I a XII) 1. b) ETN, Ab. c) Amplia difusión, epifitando a Hypnea musciformis, Cystoseira crinita, Spyridia filamentosa, Zostera marina y otras.

Enteromorpha torta (Mertens) Reinbold

a) 17 (VI a X) 1. b) -,APtf. c) Sobre Cystoseira crinita.

Ulva olivascens Dangeard

a) 6 (I a XII) 5; 17 (I a XII) 2. b) PhI, Ab.c) Forófita de Amphiroa beauvoisii. En lugares muy degradados. En el puerto forma una franja continua en el cantil vertical con ejemplares de hasta $30 \mathrm{~cm}$.

Ulva rigida C. Agardh

a) 1 (I a XII) 1; 4 (I a XII) 1. b) PhIP, C. c) Forófita de Jania rubens y Ceramium diaphanum. 


\section{CLADOPHORALES}

\section{Cladophoraceae}

Chaetomorpha aerea (Hudson) Kützing

a) 1 (I a XII) +; 6 (I a XII) +; 17 (I a XII) 1. b) RM,C. c) Epífita de Cystoseira sp.pl. e Hypnea musciformis.

Chaetomorpha linum (Müller) Kützing

a) 1 (V a VIII y I,II) 1; 17 (VI,VII,X,XI,II) 1. b) RM, C. c) Formando grandes marañas flotantes de hasta $1 \mathrm{~m}$ de diámetro. Abunda en el extremo noroeste de la laguna asociada a Cladophora vadorum.

\section{Cladophora coelothrix Kützing}

a) 4 (IV,V) 1.b) SSBc, IAo. c) Epífitas: Erythrotrichia carnea, Ceramium cingulatum, Antithamnion cruciatum y Audouinella daviesii.

Cladophora dalmatica Kützing

a) 4 (V a II) 1; 17 (IV,VIII) +. b) RMM2, Abt. c) Sobre algas del cantil vertical: Gelidium latifolium, fundamentalmente.

Cladophora echinus (Biasoletto) Kützing

a) 1 (I a XII) 1; 4 (I a XII) 1; 6 (I a XII) 1; 17 (I a XII) 1. b) PhIC, IP. c) Sobre todo sobre valvas vacías y piedras sueltas. Epífita de Cystoseira crinita y Halopteris filicina.

Cladophora globulina (Kützing) Kützing

a) 1 (I a XII) 2; 17 (I a XII)2. b) -, M. c) Forma grandes masas algodonosas sobre fondos con Caulerpa prolifera, a profundidades de hasta 3 ó $4 \mathrm{~m}$.

Cladophora lehmaniana (Lindenberg) Kützing

a) 4 (III,IV,V y VIII a XII) 1. b) SCI, Ab. c) Esciáfila.

Cladophora pellucida (Hudson) Kützing

a) 4 (VII) +. b) SSB, Ab

Cladophora prolifera (Roth) Kützing

a) 4 (V,VI,VII,IX) +. b) AS, Abt. c) Algo esciáfila. Forófita de Schmitziella endophloea.

Cladophora vadorum (Aresch.) Kützing

a) 1 (I a XII) 4; 15 (I a XII) 4; 17 (I a XII) 4; 18 (I a XII) 4. b) PhIC, -. c) Cubre grandes extensiones a ras de agua, sobre todo en los extremos de la laguna y en zonas de marismas con una degradación alta.

Cladophora vagabunda (L.) Hoek

a) 17 (IV,V,VIII,X) +. b) RM, Abt 
Rhizoclonium tortuosum (Dillwyn) Kützing

a) 1 (V,VI) 1; 4 (X)+;17 (VI) +. b) EM, C. c) Sobre el bivalvo Cardium sp.

Rhizoclonium riparium (Roth) Harvey

a) $4(\mathrm{X})+; 17(\mathrm{VIII})+$. b) ETN, Abt. c) Sobre Alsidium corallinum.

SIPHONOCLADALES

Valoniaceae

Valonia utricularis (Roth) C. Agardh

a) 1 (V a VIII) 1; 4(IX,X,XI) 1. b) SSR, P. c) En la zona 1 abundante sobre fango reductor.

\section{DASYCLADALES}

\section{Acetabulariaceae}

Acetabularia acetabulum (L.) Silva

a) 1 (IV a VIII) 1; 4 (IV a IX) 2; 17 (V,VI,VII) 1. b) PhIC, IAt. c) Bastante difundida sobre rocas horizontales fuertemente iluminadas y a menos de $1 \mathrm{~m}$ de profundidad. Asociada en muchos casos a Padina pavonica.

\section{BRYOPSIDALES}

Bryopsidaceae

Bryopsis plumosa (Hudson) C. Agardh nicaensis.

a) 4 (I a XII) 1. b) PhIP, APo. c) Esciáfila, junto a Peyssonelia squamaria y Schottera

Derbesia tenuissima (De Notaris) Crouan et Crouan

a) $4(\mathrm{X})+$. b) ETN, Ab. c) Sobre Cystoseira compressa.

Pedobesia lamourouxii (J.Ag.) J.Feld., Lor., Codomier et Couté.

a) $4(\mathrm{IV}, \mathrm{V})+$. b) HSPP, At. c) Sobre Gelidium pusillum.

\section{Codiaceae}

Codium effusum (Rafinesque) Delle Chiaje

a) 4 (IV,V,VI) +. b) SCI, IAo. c) En una zona relíctica próxima a la bocana.

Caulerpaceae

Caulerpa prolifera (Forsskaal) Lamouroux

a) 1 (I a XII) 4; 4 (I a XII) 2; 6 (I a XII) 5; 17 (I a XII) 5. b) PhIM, At. c) Esta especie ocupa la práctica totalidad de los fondos arenoso-fangosos de la Mar Chica, a partir, especialmente, de $1 \mathrm{~m}$ de profundidad. Hasta los 2,5 m cohabita con Zostera marina, a más profundidad se encuentra en solitario. Algunos epífitos como Erythrotrichia carnea y Polysiphonia elongata. 


\title{
LILIOPSIDA
}

\author{
NAJADALES
}

\section{Zosteraceae}

\section{Zostera marina L.}

a) 1(I a XII) 3; 4 (I a XII) 3; 6(I a XII) 2; 17 (I a XII) 2. b) PhIM, C. c) Multitud de epífitos, destacando Chondria tenuissima, Chondria mairei, Fosliella farinosa, Enteromorpha prolifera, Ceramium diaphanum y algunas Cladoforáceas y Ectocarpáceas. Muy difundida por fondos arenosos de menos de 2,5 m, asociada a Caulerpa prolifera y en zonas de aguas casi estancadas a Zostera noltii. Fructificada en primavera y verano.

\section{Zostera noltii Hornemann}

a) 1 (I a XII) 1. b) PhIM, Ab.c) Fructificada (V,VI).Al igual que la especie anterior con muchos epífitos, de los que destaca por su abundancia Chondria tenuissima. Recluida a zonas de muy poca profundidad donde las aguas permanecen muy estancadas.

\section{RESULTADOS Y CONCLUSIONES}

La flora bentónica de la Mar Chica es una representación simplificada de la del Mediterráneo, al igual que ocurre con la de otras lagunas en las que las condiciones morfológicas y climáticas son similares. Comparando florísticamente la Mar Chica con otras lagunas mediterráneas (Pérez- Ruzafa 1989), encontramos cierto paralelismo con gran parte de las del Mediterráneo Occidental.

El número de taxa que presentamos es de 113 (62 Rodófitas, 18 Feófitas y 31 Clorófitas, lo que implica un $\mathrm{R} / \mathrm{F}$ de 3,44; además de 2 fanerógamas). (Fig. 1). No se han podido constatar las citas de Sauvageau y R.de Buen (in Hamel 1931-1939) de Cystoseira tingitana, ni de Posidonia oceanica (Lozano op.cit.). Deducimos en este último caso la sustitución de las praderas de la fanerógama por las de Caulerpa prolifera, que aparece entremezclada y hasta $-2.5 \mathrm{~m}$ con Zostera marina en fondos de arena fangosa. Es por lo que la máxima diversidad queda reducida tan sólo a las zonas rocosas de Altos del Galán y El Atalayón.

En cuanto al origen fitogeográfico de los taxa, cabe resaltar la abundancia de elementos cosmopolitas y subcosmopolitas $(42,72 \%)$, así como los de procedencia atlántica (incluyendo los indoatlánticos y atlántico-pacíficos), con un 40\%. Los mediterráneos representan el 10\% (Fig. 2).

Teniendo en cuenta los grupos ecológicos a los que pertenecen los taxa, encontramos que el $49 \%$ son fotófilos y el 22,2\% esciáfilos. Las especies termófilas o de afinidades calientes representan el 10,1\%. Cabe además resaltar el alto grado de taxa $(37,9 \%)$ de medios calmos, portuarios y más o menos degradados, en concordancia con las condiciones físico-químicas de la laguna, lo que nos confirma un aumento del grado de eutrofización con respecto a trabajos anteriores (Lozano op.cit., Erimesco 1961). Esto puede explicar la alta presencia de taxa y, sobre todo, de poblaciones de 
las familias Ulvaceae y Cladophoraceae, especialmente en los extremos geográficos de la laguna y en su costa interna, donde la actividad humana ha sufrido un aumento considerable en las últimas décadas.

Según la fenología, los meses comprendidos entre mayo y agosto son los que presentan, tanto una mayor diversidad, como un mayor desarrollo vegetativo con una mayor presencia de órganos reproductores.(Figs. 3 y 4).

\section{BIBLIOGRAFÍA}

ATHANASIADIS, A. -1987- A survey of the seaweeds of the Aegean Sea with taxonomic studies of the tribe Antithamnieae (Rhodophyta). Akademisk avhanling University of Gothenburg Faculty of Natural Sciencies.

BOUDOURESQUE, C.F. -1984- Groupes écologiques d'algues marines et phytocenosis benthiques en Méditerranée nord-occidental: une revue. Giornale Botanico Italiano, 118:(12) suplemento $2: 7-42$.

BOUDOURESQUE, C.F. \& M. PERRET -1987- A checklist of the benthic marine algae of Corsica. GIS Posidonia publ., Marseille,121 pp.

ERIMESCO, P. -1961- La Mar Chica de Melilla. Bull. Inst. pêches marit., Maroc, 7:3-11

GIACONNE, G., P. COLONNA, C. GRAZIANO, A.M. MANNINO, E. TORNATORE, M. CORMACI, G. FURNARI \& B. SCAMMACCA -1985- Revisione della flora marina di Sicilia e isole minori. Boll.Acc. Gioenia Sci. Nat.,18 (326):537-781.

HAMEL,G. -1931/1939- Phaéophycées de France. 63 figs.+ 10 pl. + XLVII, 431 p. París

LOZANO CABO, F. -1953- Notas sobre una prospección pesquera en Mar Chica. Bol. Inst. Esp. Ocean., 64:3-37.

MAROST -1987-Documento interno. Empresa Marost.

PARKE, M. \& P.S. DIXONS Checklist of British Marine Algae. Jour. Mar. Biol. Ass. U.K., 56:527-594.

PEREZ RUZAFA, I. -1989- Fitobentos de una laguna costera. El Mar Menor. Tesis Doctoral. Univ. Murcia. 356 pp.

SILVA, P.C. \& H.W. JOHANSEN -1986- A reappraisal of the order Corallinales (Rhodophyceae). Br. Phycol. J., 21:245-254.

WYNNE,M.J. \& G.T. KRAFT -1981-.Classification summary.In C.S.Lobban \& M.J. Wynne (eds.): The biology of Sea-weeds. Bot. Monographs., 17:734-750.

YUS, R. \& J.M. CABO -1985- Guía de la Naturaleza de la zona de Melilla. Fund. Munic. Socio-Cultural. Ayunt. de Melilla.

(Aceptado para su publicación en Diciembre de 1.990)

Dirección de los autores: J.A. GONZALEZ: Escuela Universitaria de Formación del Profesorado de E.G.B. Universidad de Granada. Melilla. F. CONDE: Departamento de Biología Vegetal. Facultad de Ciencias. Universidad de Málaga. 